\title{
Self-consistent phonons: An accurate and practical method to account for anharmonic effects in equilibrium properties of general classical or quantum many-body systems.
}

\author{
Sandra E. Brown ${ }^{1}$ and Vladimir A. Mandelshtam ${ }^{1}$ \\ University of California, Irvine
}

The self-consistent phonons (SCP) method is a practical approach for computing structural and dynamical properties of a general quantum or classical many-body system while incorporating anharmonic effects. However, a convincing demonstation of the accuracy of SCP and its advantages over the standard harmonic approximation is still lacking. Here we apply SCP to classical Lennard-Jones (LJ) clusters and compare with numerically exact results. The close agreement between the two reveals that SCP accurately describes structural properties of the classical LJ clusters from zero-temperature (where the method is exact) up to the temperatures at which the chosen cluster conformation becomes unstable. Given the similarities between thermal and quantum fluctuations, both physically and within the SCP ansatz, the accuracy of classical SCP over a range of temperatures suggests that quantum SCP is also accurate over a range of quantum de Boer parameter $\Lambda=\hbar /(\sigma \sqrt{m \varepsilon})$, which describes the degree of quantum-character of the system.

\section{INTRODUCTION}

Among the variety of quantum dynamics methods which have been developed so far, any single method (or class of methods) is either approximate (inaccurate) or strictly limited in its applications (e.g., has poor numerical scaling with the system size) or both. Indeed, all suffer from significant, and in some instances crippling, limitations. For example, the exact-in-principle basis set methods scale exponentially with system size, and the real-time quantum dynamics methods based on path integrals are prohibitively expensive for systems containing more than a few degrees of freedom, due to the well-known numerical sign problem. Several other methods are restricted to the calculation of ground-state properties, e.g., diffusion Monte Carlo (DMC) ${ }^{1,2}$ and the path-integral ground state (PIGS) method ${ }^{3}$. Imaginarytime path integral Monte Carlo (PIMC) is routinely used to treat quantum many-body systems, but are limited to calculations of equilibrium properties at finite temperatures. Two popular ad hoc quantum dynamics methods based on path integrals, centroid molecular dynamics $(\mathrm{CMD})^{4}$ and ring-polymer molecular dynamics $(\mathrm{RPMD})^{5,6}$ are known to produce unphysical artifacts, making them a poor choice in many instances, in particular, for calculation of spectra. Perturbation theory for a many-body system is often unreliable and complicated, both numerically and in determining whether it is even suitable for the system at hand. At the other extreme, the harmonic approximation (HA) is unique in its ability to provide an essentially complete description of a quantum many-body system with a given potential energy for a large class of systems and properties, without requiring explicit simulation. However, this comes at the price of working with a method which lacks a reliable and practical procedure to either assess its accuracy or improve it in a systematic fashion by including anharmonic corrections. Noteworthy attempts to improve upon the harmonic approximation include a perturbative approach ${ }^{7}$ that strives to incorporate anharmonic effects by working with temperature-dependent frequencies. While this method does capture some anharmonic effects, it is also limited in some aspects, such as its ability to account for structural changes (such as cluster expansion) due to thermal fluctuations.

The self-consistent phonons (SCP) method offers a practical way to improve the HA for computing structural, thermodynamic and dynamical properties of a general, classical or quantum, many-body system. For a system at thermal equilibrium in a basin of attraction, this variational approach yields the "best" temperaturedependent harmonic approximation to the potential energy surface (PES), that is, it gives the effective harmonic potential which minimizes the Helmholtz free energy of the system at a given temperature. In this way, anharmonic effects are naturally incorporated. SCP was first introduced several decades ago as a means to include anharmonic effects in the approximate treatment of the nuclear dynamics of condensed phase systems ${ }^{8,9}$. The idea of replacing the physical system by a temperaturedependent effective harmonic system is appealing, and had previously been exploited by at least one alternative approach, principal mode analysis (PMA $)^{10}$, and has been rediscovered at least once ${ }^{11}$. Interest in SCP in the context of finite systems has emerged only recently. In Ref. 12, SCP was used to compute the fundamental frequencies of aromatic hydrocarbons, and in Refs. 13 and 14, for computing the ground states of very large LJ clusters. Given a quantum $N$-body system localized in an energy minimum at thermal equilibrium, SCP maps it to a reference temperature-dependent harmonic system by optimizing the free energy in accordance with the Gibbs-Bogoliubov variational principle. The key numerical bottleneck of the method is then associated with numerical evaluation of Gaussian-weighted multivariate integrals all having the reduced form

$$
\langle f(\mathbf{x})\rangle=\int_{\mathbb{R}^{3 N}} \exp \left(-\frac{1}{2} \mathbf{x}^{\mathrm{T}} \mathbf{x}\right) f(\mathbf{x}) d \mathbf{x},
$$


with $f(\mathbf{x})$ sufficiently smooth for a numerical solution to be obtained by way of Monte Carlo integration. For pairwise potentials these integrals can be computed very efficiently $^{15}$, which has been utilized within the SCP framework in Ref. 14 for Lennard-Jones (LJ) clusters consisting of many thousands of atoms. However, for a general case, when an efficient parametrization of the system potential cannot be obtained, the numerical integration may be extremely expensive. As an example, a "naive" approach based on standard Monte Carlo (MC) results in SCP being even more expensive than RPMD when applied to hydrocarbons described by a tight-binding model $^{12}$, due to the slow $O\left(1 / \sqrt{N_{\mathrm{MC}}}\right)$ scaling of the statistical error in the MC integration, where $N_{\mathrm{MC}}$ is the number of MC points. In Ref. 16 we have shown how the integration can be performed numerically for a general potential most efficiently using quasi-MC, which exhibits a superior scaling of the integration error $\sim O\left(1 / N_{\mathrm{MC}}\right)$. This approach, applied to the same aromatic hydrocarbons as in Ref. 12 reduced the computational cost of SCP by several orders of magnitude, expanding its range of applicability to $a b$ initio potentials. Ref. 16 also reports SCP fundamental frequencies for $\mathrm{OH}$ stretching and $\mathrm{HOH}$ bending modes in water hexamer, which did show the physically correct trends in terms of the anharmonic contributions. At the same time, the SCP results were in significant quantitative and qualitative disagreement with the results of Wang and Bowman using the local monomer model (LMM) ${ }^{17}$. Unfortunately, currently no method exists that could reliably predict such quantities for a system as complex as quantum water hexamer, making the assessment of the accuracy of a quantum dynamics method applied to a general many-body system a critical issue. As is the case with most other quantum many-body approaches, the SCP approximation is uncontrollable in the sense that there is generally no consistent procedure to systematically improve its accuracy by a gradual increase of the computational effort. That is, in order to go beyond the SCP approximation one needs to make a heroic effort. (See Refs. 11 and 18 for examples of such extensions.)

To reiterate, SCP is inherently approximate, and an empirical/numerical assessment of its accuracy is crucial. Incidentally, some evidence of the accuracy of the method has already been established. As discussed in Ref. 18, $\mathrm{SCP}$ is equivalent to the variational Gaussian wavepacket (VGW) $\operatorname{method}^{19,20}$ in the $T \rightarrow 0$ limit. Ref. 21 demonstrated very good agreement between VGW ground state energies of several isomers of $\mathrm{Ne}_{38}$ with those obtained from low-temperature path integral Monte Carlo (PIMC) calculations. In addition, Ref. 19 demonstrated agreement between VGW and PIMC at very low temperature for the pair correlation function of $\mathrm{Ne}_{13}$.

In this paper we carry out an assessment of the accuracy of SCP for the calculation of equilibrium structural properties for a classical system at finite temperatures. In particular, we consider the radial distribution function and principal moments of inertia for LJ clusters. Com- puted equilibrium structures are clearly of great interest and utility in their own right. Additionally, the accurate determination of these structural properties makes possible the comparison between theoretical and experimental results. The link between the radial distribution function $g(r)$ and the structure factor determined in scattering experiments, and between the principal moments of inertia and rotational spectra makes it possible to combine theoretical and experimental avenues of inquiry. Considering the analogous treatment of quantum fluctuations and thermal fluctuations in the SCP framework, the accuracy of the method for a classical system over a given range of temperatures suggests that it should also be accurate for systems which do not exhibit too much quantum character. As will be discussed, it should be considered one of the key advantages of the SCP method over other approaches that its implementation is virtually the same for both classical and quantum systems.

LJ clusters have long been a popular system for testing sampling and optimization algorithms/methods and for studying fundamental physical phenomena. The LJ pair potential is given by

$$
V(\mathbf{r})=4 \varepsilon\left[\left(\frac{\sigma}{r}\right)^{12}-\left(\frac{\sigma}{r}\right)^{6}\right],
$$

where the energy scale and the length scale are defined by the LJ parameters, i.e., the well-depth, $\varepsilon$, and the interparticle distance at which the potential is zero, $\sigma$. For systems governed by this potential, the degree of quantum delocalization can be conveniently described by the quantum de Boer parameter,

$$
\Lambda:=\hbar /(\sigma \sqrt{m \varepsilon}) .
$$

Note that $\Lambda=0$ corresponds to a classical system, and $\Lambda \approx 0.095$ to neon. Though governed by a simple potential, LJ clusters exhibit rich behavior and properties, which are by now very well understood. The comprehensive body of theoretical work for these clusters includes studies of their potential energy landscapes, and phase/structural transitions induced by changes in size, temperature, or quantum parameter. (See, e.g., Refs. 2231 , and references therein.) Their use in the present work enables us to reliably demonstrate the accuracy of the SCP method, without risking the introduction of unnecessary complications arising from any poorly understood or pathological behavior of the potential or system used. Moreover, the de Boer parameter provides the desired well-defined and quantifiable degree of quantum character for this potential.

In contrast to methods such as replica-exchange MC/MD (or replica exchange PIMC/PIMD) in which many minima are sampled, both the standard HA and SCP break down once the simulation leaves the basin of attraction. For this reason, comparisons of equilibrium thermodynamic quantities obtained using these two different types of methods are generally not meaningful at temperatures where multiple isomers or minima contribute. With this in mind, we have chosen for the sake 
of simplicity to consider the minimum energy isomers of the $\mathrm{LJ}_{13}, \mathrm{LJ}_{38}$, and $\mathrm{LJ}_{55}$ clusters, as all three exhibit "deep" global minima separated from other (local) minima by large barriers. Here we assess the accuracy of SCP for a classical system at finite temperature by comparing to Markov chain Monte Carlo (MC) using the wellknown Metropolis-Hastings algorithm. This method is exact in the sense that the computed ensemble averages are guaranteed to converge to the true values for a longenough simulation, and is also exceptionally simple to implement, even in the present case where we wish to sample only a particular minimum of the PES.

\section{SELF-CONSISTENT PHONONS}

Given a (classical or quantum) many-body system at thermal equilibrium in a basin of attraction, the SCP method gives the temperature-dependent, effective harmonic Hamiltonian which minimizes the Helmholtz free energy of the system,

$$
H_{h}(T)=-\frac{\hbar^{2}}{2} \nabla^{\mathrm{T}} \mathbf{M}^{-1} \nabla+\frac{1}{2}(\mathbf{r}-\mathbf{q})^{\mathrm{T}} \mathbf{K}(\mathbf{r}-\mathbf{q})+V_{0},
$$

where $T$ is the temperature of the system; $\mathbf{M}$ is the mass matrix; and $V_{0}, \mathbf{q}$ and $\mathbf{K}$ are the minimum, center, and Hessian of the effective harmonic potential, respectively. This is achieved by invoking the Gibbs-Bogoliubov variational principle

$$
F \leq F_{\text {trial }}:=F_{h}+\langle V\rangle_{h}-\left\langle V_{h}\right\rangle_{h},
$$

with $\mathbf{q}$ and $\mathbf{K}$ as variational parameters, which gives rise to the SCP equations

$$
\begin{aligned}
& \langle\nabla V\rangle_{h}=0 \\
& \left\langle\nabla \nabla^{\mathrm{T}} V\right\rangle_{h}=\mathbf{K} .
\end{aligned}
$$

Note that the ensemble averages $\langle\cdot\rangle_{h}$ are taken with respect to the effective harmonic system:

$$
\begin{aligned}
\langle f(\hat{\mathbf{r}})\rangle_{h} & :=\operatorname{Tr}\left[e^{-\beta \hat{H}_{h}} f(\hat{\mathbf{r}})\right] \\
& =\|2 \pi \mathbf{D}\|^{-1 / 2} \int e^{-\frac{1}{2} \mathbf{X}^{\mathrm{T}} \mathbf{D}^{-1} \mathbf{x}} f(\mathbf{q}+\mathbf{x}) d \mathbf{x},
\end{aligned}
$$

where the displacement-displacement correlation matrix $D$ is given by

$$
\mathbf{D}=\mathbf{M}^{-1 / 2} d(\boldsymbol{\Omega}) \mathbf{M}^{-1 / 2},
$$

with the frequency matrix $\boldsymbol{\Omega}$ defined via the mass-scaled effective Hessian,

$$
\boldsymbol{\Omega}^{2}=\tilde{\mathbf{K}}=\mathbf{M}^{-1 / 2} \mathbf{K M}^{-1 / 2},
$$

and the auxiliary function $d$ defined by

$$
d(\omega):=(\hbar / 2) \omega^{-1} \operatorname{coth}(\beta \hbar \omega / 2) .
$$

The above set of coupled, nonlinear differential equations (2) can be solved iteratively at each $T$ or $\Lambda$, e.g., using a Newton-Raphson type approach,

$$
\begin{aligned}
\mathbf{D}^{\text {(next) }} & =\mathbf{M}^{-1 / 2} d(\boldsymbol{\Omega}) \mathbf{M}^{-1 / 2} \\
\mathbf{K}^{\text {(next) }} & =\left\langle\nabla \nabla^{\mathrm{T}} V\right\rangle_{h} \\
\mathbf{q}^{\text {(next) }} & =\mathbf{q}-\tau \mathbf{D}\langle\nabla V\rangle_{h},
\end{aligned}
$$

where $\tau$ is a scalar, whose value can be optimized to achieve the fastest convergence while still maintaining the stability of the iterative process.

Note that the Hessian, $\nabla \nabla^{\mathrm{T}} V$, does not need to be computed explicitly in the SCP ansatz ${ }^{16}$, as a consequence of working with Gaussian integrals. Instead, we may consider the average product of the displacement and the gradient,

$$
\left\langle\nabla \nabla^{T} V(\mathbf{r})\right\rangle_{h}=\mathbf{D}^{-1}\left\langle(\mathbf{r}-\mathbf{q})^{T} \nabla V(\mathbf{r})\right\rangle .
$$

This property circumvents the enormous expense associated with computing an analytic Hessian, supposing it is even available, or a finite-difference Hessian when it is not. While this "trick" generally comes at the price of working with an integrand which is more oscillatory, and hence an integral which converges more slowly, the overall reduction in computational cost is still substantial, especially when using more complex or ab initio potentials.

The simple form of the Lennard-Jones potential and its low computational cost affords us the freedom to choose any of the three variations mentioned, i.e., exploit Eq. 8 and avoid computing the Hessian altogether, compute a finite-difference Hessian, or compute the analytic Hessian. The results presented in this work were obtained from SCP simulations which made use of the analytic Hessian. (All three variations were implemented in preliminary simulations and produced identical results.) Earlier work $^{14}$ using SCP to study thermal and quantum transitions in large LJ clusters $\left(N \sim 10^{4}\right)$ made use of the ability to fit the LJ pair-potential by a sum of Gaussians ${ }^{15}$. However, the improved efficiency of the SCP method with the incorporation of quasi-Monte Carlo integration ${ }^{16}$ and comparatively modest cluster sizes examined here preclude the need for such fitting of the potential.

One of the distinct features of SCP is that its implementation is virtually the same for classical and quantum systems, with no additional overhead required for the quantum case. Ref. 14 considers two important limits of SCP: the zero-temperature $(T=0)$ limit, and the classical $(\Lambda=0)$ limit, and shows that the SCP equations in each limit can be formulated in terms of the solution of a system of ordinary differential equations along a fictitious time, with the two systems having remarkably similar structure. (For the quantum case in the zero-temperature limit, SCP turns out to be equivalent to the variational Gaussian wavepacket (VGW) approximation ${ }^{19,20}$; in both cases the quantum ground 
state constrained to a local potential energy minimum is approximated by the same variational Gaussian.) This resemblance of the classical $(\Lambda=0, T>0)$ and quantum $(T=0, \Lambda>0)$ limits seems to offer the possibility of establishing an almost quantitative relationship between the quantum parameter $\Lambda$ and temperature $T$. In the present context, this relationship will be used as an argument to justify the utility of the SCP method for the quantum case over a range of quantum parameters $\Lambda$ based on its favorable assessment in the classical limit over a range of temperatures.

\section{NUMERICAL RESULTS}

HA, SCP, and MC calculations were carried out beginning from the minimum energy configurations obtained from the Cambridge Cluster Database ${ }^{32}$. SCP simulations for the classical $\mathrm{LJ}_{13}, \mathrm{LJ}_{38}$, and $\mathrm{LJ}_{55}$ clusters typically used 20 iterations at each temperature. Between 20 and 100 iterations were carried out at each value of $\Lambda$ for the quantum $\mathrm{LJ}_{13}, \mathrm{LJ}_{38}$, and $\mathrm{LJ}_{55}$ clusters. For the $\mathrm{Ne}_{13}$, $\mathrm{Ne}_{38}$, and $\mathrm{Ne}_{55}$ clusters, 100 iterations were carried out at each temperature. At each iteration, $S=2^{15}$ Sobol points were used to compute the average potential, gradient, and Hessian with respect to the reference harmonic system. Based on preliminary SCP calculations employing Sobol sequences of various lengths, this value of $S$ was chosen to exclude any ambiguity associated with the numerical accuracy of computed integrals. On the final iteration for each temperature $T$ or quantum parameter $\Lambda$, the $2^{15}$ Sobol points were used to average the radial distribution function and principal moments of inertia. In order to maintain a fair comparison of the methods, for the case of HA the structural properties were computed using the same protocol as that for SCP with the only difference being the different harmonic Hamiltonians used to compute the averages.

MC simulations were carried out from low temperature (0.001 $\left.\varepsilon / k_{B}\right)$ up to roughly the temperatures at which the random walk would become unstable (i.e., would escape from the potential energy minimum, in which it was initialized) with temperature increments of either 0.001 $\varepsilon / k_{B}$ or $0.010 \varepsilon / k_{B}$, and $10^{8}$ trial MC moves completed at each temperature. (Each trial MC move is an attempt to displace a single particle in the Metropolis algorithm.) Hard constraining radii of $2.0 \sigma, 3.0 \sigma$, and $3.5 \sigma$ (with respect to the center of mass) were used for $\mathrm{LJ}_{13}, \mathrm{LJ}_{38}$, and $\mathrm{LJ}_{55}$, respectively.

The radial pair correlation functions $p(r / \sigma)$ for classical LJ ${ }_{n}$ clusters $(n=13,38,55)$ computed by three methods (HA, SCP, and MC) are shown in figures 1 through 3. At low temperature $\left(T=0.056 \varepsilon / k_{B}\right) \mathrm{HA}, \mathrm{SCP}$, and $\mathrm{MC}$ all agree well with one another, as one would expect. However, close inspection of individual peaks and troughs in the pair correlation functions reveals that even at this low temperature, HA already deviates slightly from the exact MC result, while SCP very faithfully adheres to it. The deviations among the three results become more noticeable as the temperature increases, still, SCP consistently produces the correct $r / \sigma$ values for the peak centroids (i.e., the mass centers) of the pair correlation functions for all three clusters considered.

Not only is HA quantitatively inaccurate, it is also intrinsically unable to produce much of the qualitatively correct physical behavior of the clusters. For example, due to the center of the harmonic potential being fixed at the minimum in HA, the peaks in the radial distribution function are unable to shift with increasing temperature or de Boer parameter. By contrast, in the SCP approximation the center and width of the effective harmonic potential are temperature- and quantum parameter-dependent, so that the peaks in the distribution function expand outward with increasing temperature and/or quantum parameter, as they should. This striking difference between the two approximations is clearly illustrated in Fig. 4, which shows the evolution of the $\mathrm{LJ}_{13}$ radial distribution function with temperature. Similarly, Fig. 5 shows the evolution of the radial distribution function with $\Lambda$ for the ground states $(T=0)$ of $\mathrm{LJ}_{13}, \mathrm{LJ}_{38}$, and $\mathrm{LJ}_{55}$. This result is another demonstration of the SCP approximation being able to correctly account for anharmonic effects corresponding to cluster expansion caused by quantum fluctuations.

As previously mentioned, Ref. 19 reports a comparison of presumably exact PIMC results ${ }^{33}$ for the radial pair correlation functions of $\mathrm{Ne}_{13}$ with those computed by VGW. Fig. 6 here shows a similar comparison of PIMC with SCP at low temperature $(T=2 \mathrm{~K}$ or $T=0.056 \varepsilon / k_{B}$ in reduced units). Though SCP and VGW are algorithmically very different, at this temperature they yield nearly the same result, which also agrees well with PIMC. Again, the standard HA does not account for cluster expansion due to the quantum fluctuations, which is manifested in the peaks of $p(r)$ maintaining significantly lower values compared to the exact result.

Finally, Fig. 7 shows the dependence of the principal moments of inertia of the same three clusters on either temperature or de Boer parameter. Again, three special cases are considered, corresponding to the classical $(\Lambda=0)$ LJ clusters (left column), quantum ground states $(T=0)$ of the quantum LJ clusters (center column), and neon $(\Lambda=0.095)$ (right column). Notably, the moments of inertia for the classical clusters computed by SCP not only display the physically correct behavior, but are also in a very good quantitative agreement with the numerically exact $\mathrm{MC}$ results up to relatively high temperatures. This is in contrast with the HA approximation, which shows physically incorrect behavior caused by the inability of HA to account for cluster expansion caused by either thermal or quantum fluctuations. Although numerically exact results are not available for the quantum LJ clusters, we believe that the SCP results are at least qualitatively correct. Again, this is supported by documented comparisons of SCP with numerically exact data 
that is available ${ }^{14,18,20,21}$, as well as the argument ${ }^{14}$ that the SCP equations for the classical and quantum cases are virtually the same, with quantum fluctuations and thermal fluctuations playing the same role and leading to the same effects, so that SCP should perform similarly when applied to the same system in either case. That is, given a classical anharmonic system $\left(\mathrm{LJ}_{n}\right.$ in the present case) for which SCP is accurate over a certain temperature range, there should also be a range of quantum parameter $\Lambda$ for which SCP accurately describes the quantum analogue of the system.

\section{CONCLUSIONS}

In this paper we presented several numerical examples demonstrating the utility of SCP as a practical, numerically inexpensive, and often quantitatively accurate method to compute equilibrium properties of general quantum many-body systems. The method is able to account for anharmonic effects, e.g., effects that cannot be captured by the standard HA. In this work we focused on the effects of temperature and quantum character on the structural properties of LJ clusters, in particular the radial pair correlation function and moments of inertia. While most comparisons of SCP with exact methods have been made with respect to classical systems, we expect that SCP should also be able to provide accurate results for quantum systems as well, based on the well-known parallel between thermal and quantum fluctuations. Finally, although SCP is an inherently approximate approach, its superiority to the standard HA, while still being significantly less numerically expensive than the exact-in-principle methods, makes it a practical compromise between HA and the much more sophisticated and/or expensive methods for computing equilibrium structural properties. In this regard it is worth metioning the harmonic superposition approach ${ }^{28}$, in which the basic element, i.e., the contrinution of each local minimum to the equilibrium properties of the system at thermal equilibrium using the standard HA could be replaced by the SCP approximation.

\section{ACKNOWLEDGEMENTS}

This work was supported by the National Science Foundation (NSF) Grant No. CHE-1566334.

\footnotetext{
${ }^{1}$ James B. Anderson, A random-walk simulation of the Schrödinger equation: $\mathrm{H}_{+}{ }^{3}$, The Journal of Chemical Physics 63 (1975), no. 4, 1499-1503.

${ }^{2}$ James B. Anderson, Quantum chemistry by random walk. $\mathrm{H}^{2} P$, $\mathrm{H}_{3}^{+} D_{3 h}{ }^{1} A_{1}^{\prime}, \mathrm{H}_{2}{ }^{3} \Sigma_{u}^{+}, \mathrm{H}_{4}{ }^{1} \Sigma_{g}^{+}, \mathrm{Be}^{1} S$, The Journal of Chemical Physics 65 (1976), no. 10, 4121-4127.

${ }^{3}$ A. Sarsa, K. E. Schmidt, and W. R. Magro, A path integral ground state method, The Journal of Chemical Physics 113 (2000), no. 4, 1366-1371.
}

${ }^{4}$ Jianshu Cao and Gregory A. Voth, A new perspective on quantum time correlation functions, The Journal of Chemical Physics 99 (1993), no. 12, 10070-10073.

${ }^{5}$ Ian R. Craig and David E. Manolopoulos, Quantum statistics and classical mechanics: Real time correlation functions from ring polymer molecular dynamics, The Journal of Chemical Physics 121 (2004), no. 8, 3368-3373.

${ }^{6}$ Bastiaan J. Braams and David E. Manolopoulos, On the short-time limit of ring polymer molecular dynamics, The Journal of Chemical Physics 125 (2006), no. 12, 124105.

${ }^{7}$ F. Calvo, J. P. K. Doye, and D. J. Wales, Characterization of anharmonicities on complex potential energy surfaces: Perturbation theory and simulation, The Journal of Chemical Physics 115 (2001), no. 21, 9627-9636.

${ }^{8}$ Thomas R. Koehler, Theory of the Self-Consistent Harmonic Approximation with Application to Solid Neon, Phys. Rev. Lett. $\mathbf{1 7}(1966), 89-91$.

${ }^{9}$ N. S. Gillis, N. R. Werthamer, and T. R. Koehler, Properties of Crystalline Argon and Neon in the Self-Consistent Phonon Approximation, Phys. Rev. 165 (1968), 951-959.

${ }^{10}$ Bernard R. Brooks, Dušanka Janežič, and Martin Karplus, Harmonic analysis of large systems. I. Methodology, Journal of Computational Chemistry 16 (1995), no. 12, 1522-1542.

${ }^{11}$ Jianshu Cao and Gregory A. Voth, Modeling physical systems by effective harmonic oscillators: The optimized quadratic approximation, The Journal of Chemical Physics 102 (1995), no. 8, 3337-3348.

${ }^{12}$ F. Calvo, P. Parneix, and N.-T. Van-Oanh, Finite-temperature infrared spectroscopy of polycyclic aromatic hydrocarbon molecules. II. Principal mode analysis and self-consistent phonons, The Journal of Chemical Physics 133 (2010), no. 7, 074303 .

${ }^{13}$ Ionut Georgescu and Vladimir A. Mandelshtam, A fast variational gaussian wavepacket method: Size-induced structural transitions in large neon clusters, The Journal of Chemical Physics 135 (2011), no. 15, 154106.

${ }^{14}$ Ionut Georgescu and Vladimir A. Mandelshtam, Self-consistent phonons revisited. I. The role of thermal versus quantum fluctuations on structural transitions in large Lennard-Jones clusters, The Journal of Chemical Physics 137 (2012), no. 14, 144106 .

${ }^{15}$ N. Corbin and K. Singer, Semiclassical molecular dynamics of wave packets, Molecular Physics 46 (1982), no. 3, 671-677.

16 Sandra E. Brown, Ionuţ Georgescu, and Vladimir A. Mandelshtam, Self-consistent phonons revisited. II. A general and efficient method for computing free energies and vibrational spectra of molecules and clusters, The Journal of Chemical Physics 138 (2013), no. 4, 044317.

${ }^{17}$ Yimin Wang and Joel M. Bowman, Ab initio potential and dipole moment surfaces for water. II. Local-monomer calculations of the infrared spectra of water clusters, The Journal of Chemical Physics 134 (2011), no. 15.

${ }^{18}$ Ionut Georgescu, Svetlana Jitomirskaya, and Vladimir A. Mandelshtam, On the ground state calculation of a many-body system using a self-consistent basis and quasi-Monte Carlo: An application to water hexamer, The Journal of Chemical Physics 139 (2013), no. 20, 204104.

${ }^{19}$ Pavel Frantsuzov, Arnold Neumaier, and Vladimir A. Mandelshtam, Gaussian resolutions for equilibrium density matrices, Chemical Physics Letters 381 (2003), no. 1-2, $117-122$.

${ }^{20}$ Pavel A. Frantsuzov and Vladimir A. Mandelshtam, Quantum statistical mechanics with Gaussians: Equilibrium properties of van der Waals clusters, The Journal of Chemical Physics 121 (2004), no. 19, 9247-9256.

${ }^{21}$ Cristian Predescu, Pavel A. Frantsuzov, and Vladimir A. Mandelshtam, Thermodynamics and equilibrium structure of $\mathrm{Ne}_{38}$ cluster: Quantum mechanics versus classical, The Journal of Chemical Physics 122 (2005), no. 15.

${ }^{22}$ J. A. Northby, Structure and binding of Lennard-Jones clusters: $13 \leq N \leq 147$, The Journal of Chemical Physics 87 (1987), 
no. $10,6166-6177$

${ }^{23}$ Jonathan P. K. Doye and David J. Wales, Calculation of thermodynamic properties of small Lennard-Jones clusters incorporating anharmonicity, The Journal of Chemical Physics 102 (1995), no. 24, 9659-9672.

${ }^{24}$ Charusita Chakravarty, Quantum delocalization and cluster melting, The Journal of Chemical Physics 103 (1995), no. 24, 10663-10668.

${ }^{25}$ Robert H. Leary, Global Optima of Lennard-Jones Clusters, Journal of Global Optimization 11 (1997), no. 1, 35-53.

${ }^{26}$ J. P. Neirotti, F. Calvo, David L. Freeman, and J. D. Doll, Phase changes in 38-atom Lennard-Jones clusters. I. A parallel tempering study in the canonical ensemble, The Journal of Chemical Physics 112 (2000), no. 23, 10340-10349.

${ }^{27}$ F. Calvo, J. P. Neirotti, David L. Freeman, and J. D. Doll, Phase changes in 38-atom Lennard-Jones clusters. II. A parallel tempering study of equilibrium and dynamic properties in the molecular dynamics and microcanonical ensembles, The Journal of Chemical Physics 112 (2000), no. 23, 10350-10357.
${ }^{28}$ F. Calvo, J. P. K. Doye, and D. J. Wales, Quantum partition functions from classical distributions: Application to rare-gas clusters, The Journal of Chemical Physics 114 (2001), no. 17, 7312-7329.

${ }^{29}$ Jonathan P. K. Doye and Florent Calvo, Entropic effects on the structure of Lennard-Jones clusters, The Journal of Chemical Physics 116 (2002), no. 19, 8307-8317.

${ }^{30}$ Vladimir A. Mandelshtam and Pavel A. Frantsuzov, Multiple structural transformations in Lennard-Jones clusters: Generic versus size-specific behavior, The Journal of Chemical Physics 124 (2006), no. 20

${ }^{31}$ Jason Deckman and Vladimir A. Mandelshtam, Effects of Quantum Delocalization on Structural Changes in Lennard-Jones Clusters ${ }^{\dagger}$, The Journal of Physical Chemistry A 113 (2009), no. 26, 7394-7402.

${ }^{32}$ D. J. Wales, J. P. K. Doye, A. Dullweber, M. P. Hodges, F. Y. Naumkin, F. Calvo, J. Hernández-Rojas, and T. F. Middleton, The Cambridge Cluster Database, http://wwwwales.ch.cam.ac.uk/CCD.html.

${ }^{33}$ Victoria Buch, Exploration of multidimensional variational Gaussian wave packets as a simulation tool, The Journal of Chemical Physics 117 (2002), no. 10, 4738-4750. 


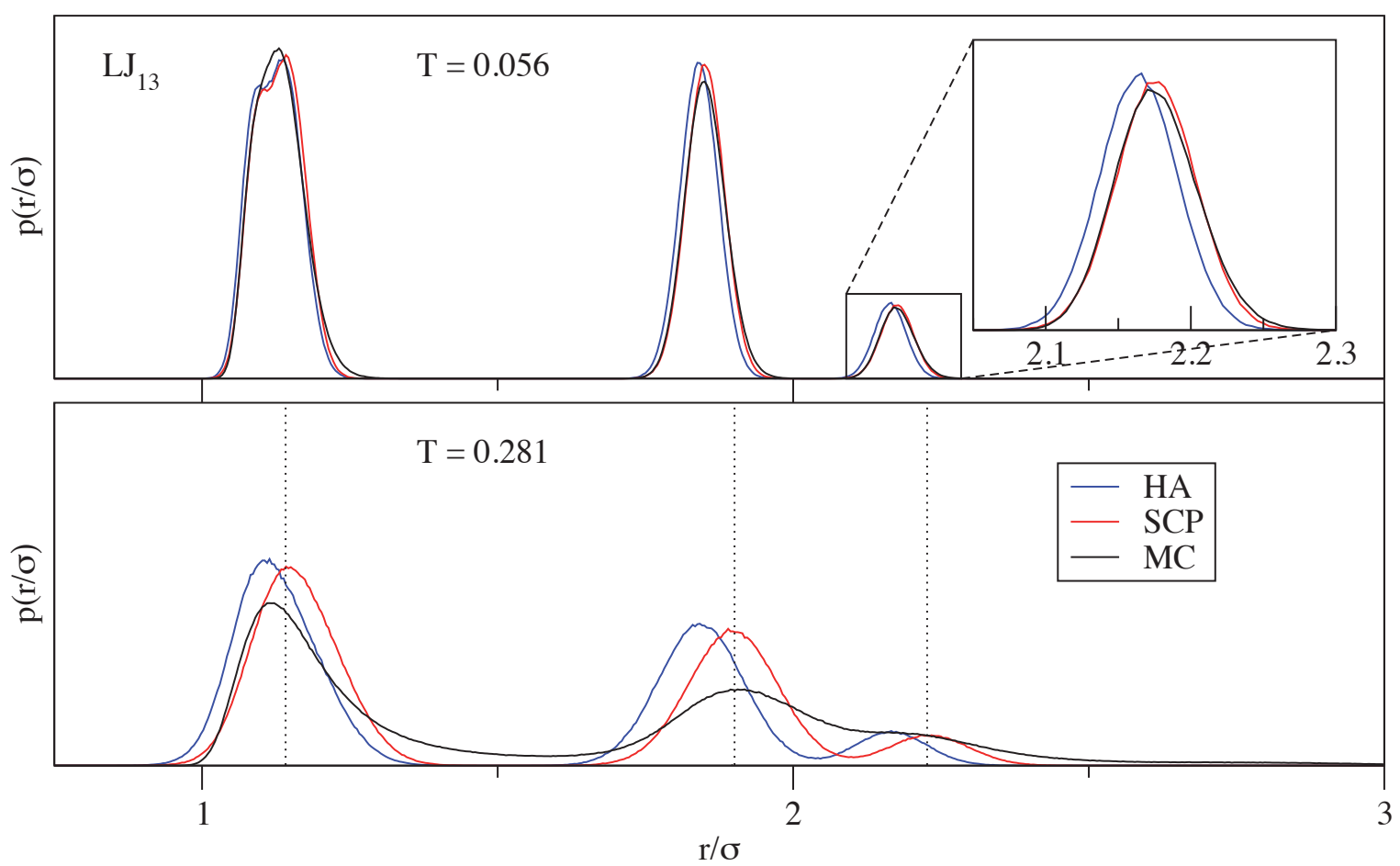

FIG. 1. Pair correlation functions for the classical $\mathrm{LJ}_{13}$ cluster at a low and high temperatures $\left(T=0.056,0.281 \varepsilon / k_{B}\right)$ computed by three different methods: the harmonic approximation (HA), the self-consistent phonons (SCP) method, and Monte Carlo (MC). The vertical dotted lines in the bottom plot indicate the positions of the peak centroids in the SCP approximation.

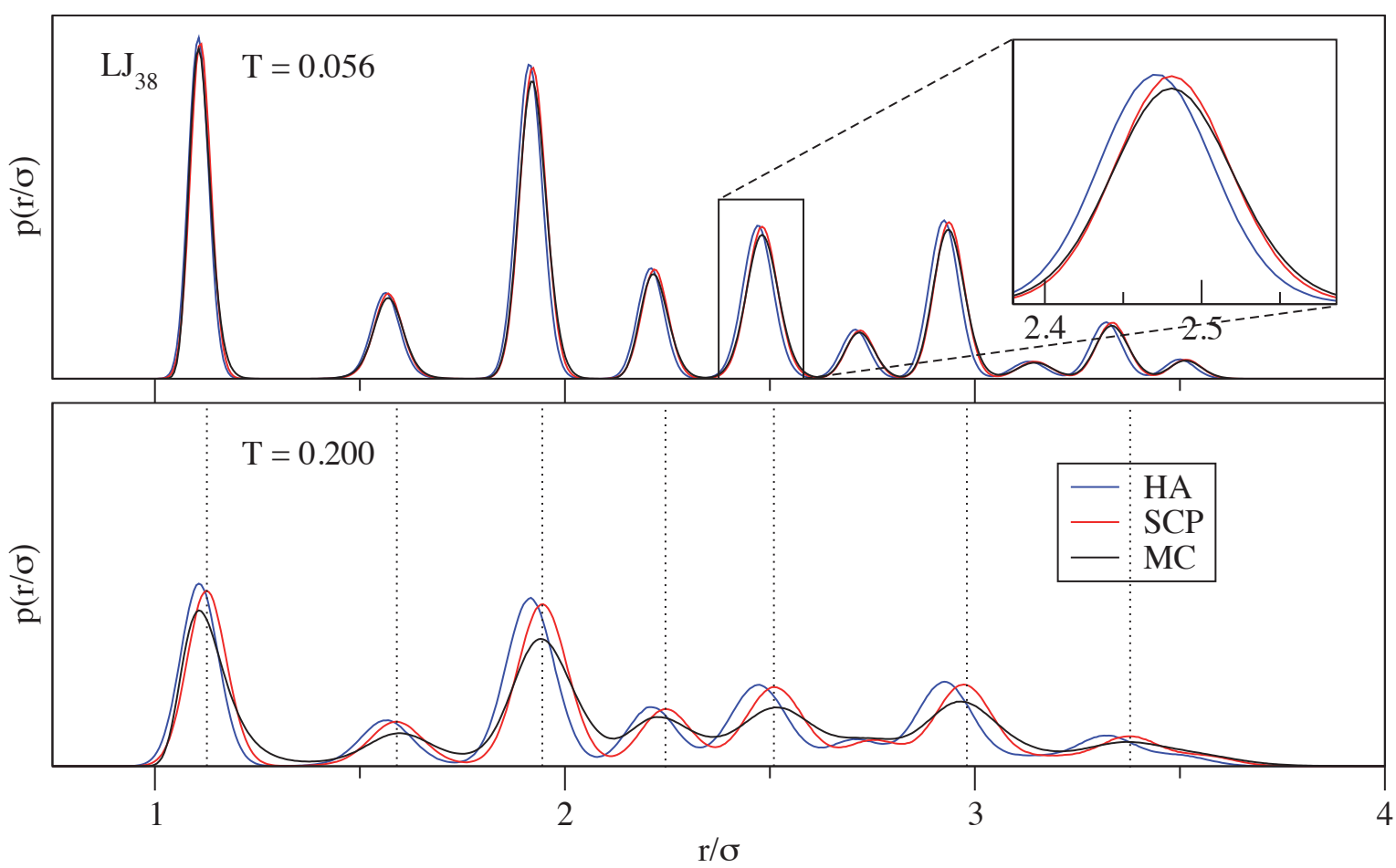

FIG. 2. Same as Fig. 1, but for $\mathrm{LJ}_{38}$. The "high temperature" $\left(T=0.200 \varepsilon / k_{B}\right)$ in this case is the temperature above which the random walk in the Metropolis Monte Carlo simulation escapes from the Oh potential energy minimum. 


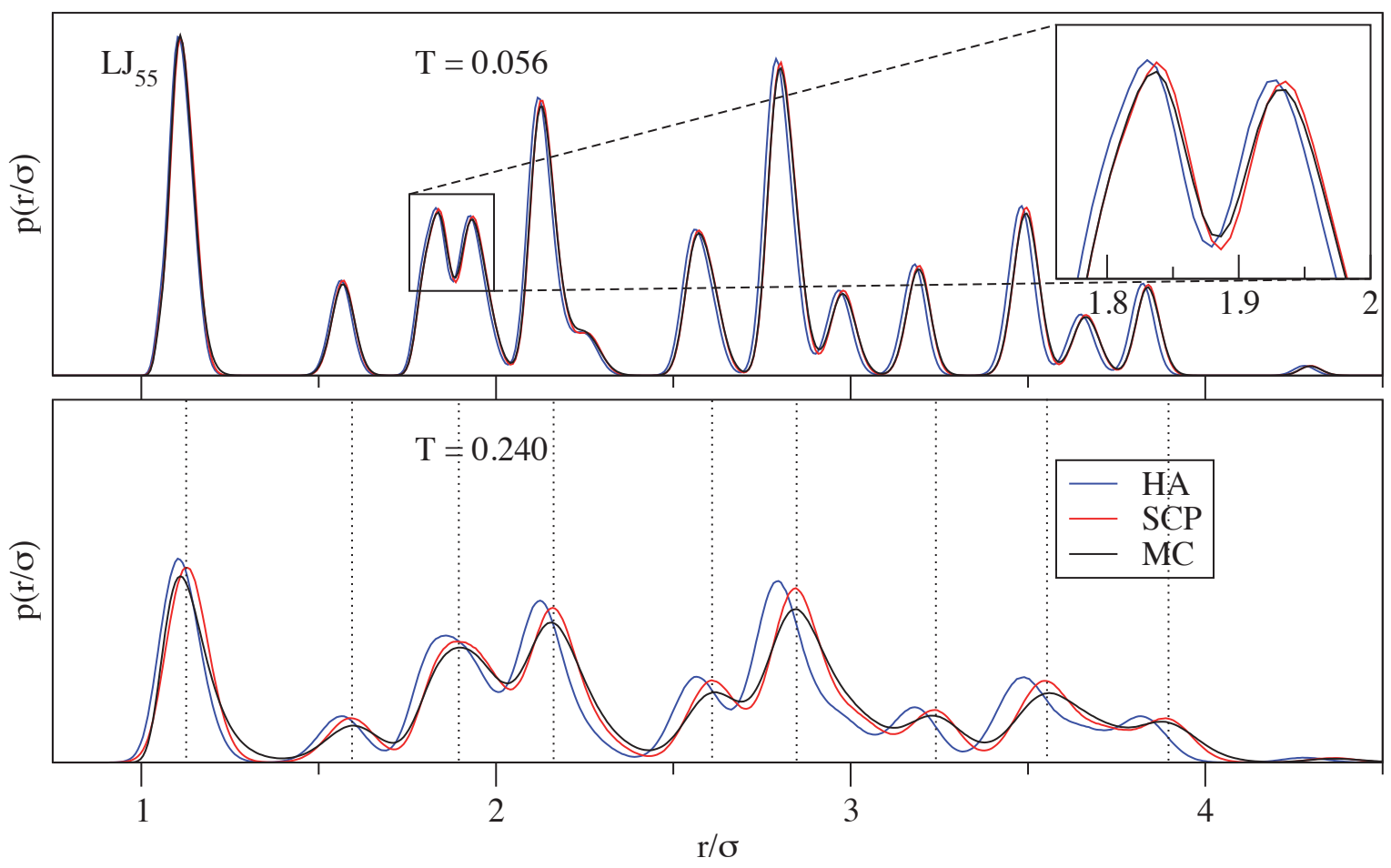

FIG. 3. Same as Fig. 1, but for $\mathrm{LJ}_{55}$ and a "high temperature" of $T=0.240 \varepsilon / k_{B}$.
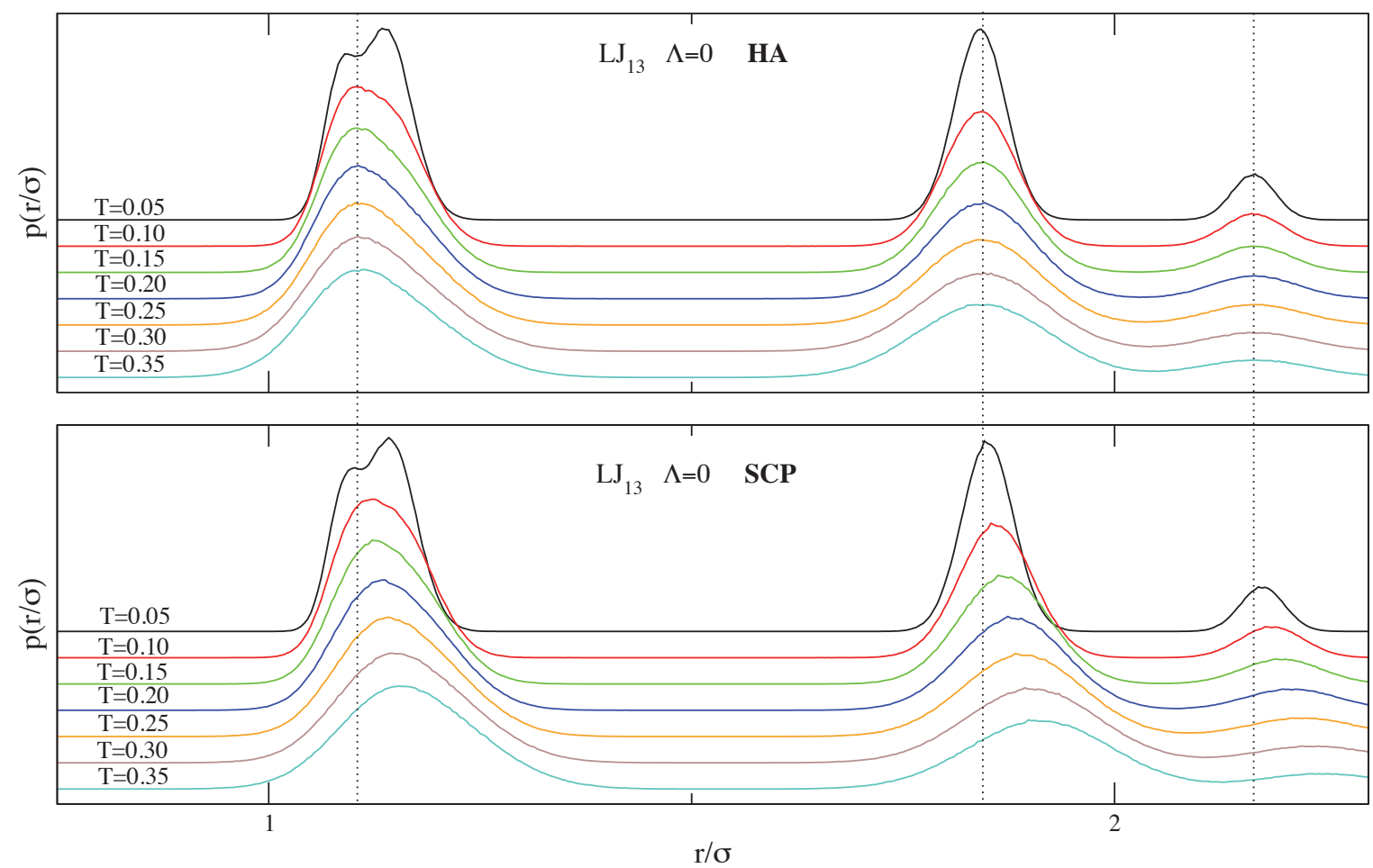

FIG. 4. Evolution of the radial distribution function of classical $\mathrm{LJ}_{13}$ with temperature, computed using the standard harmonic approximation (HA) and self-consistent phonons (SCP) method. Note that the SCP method is able to produce both the broadening and drifting of the peaks with temperature, while in the HA the maxima do not shift with temperature. The vertical dotted lines are included to highlight the discrepancy between the locations of the peak centroids in the HA and SCP results. 

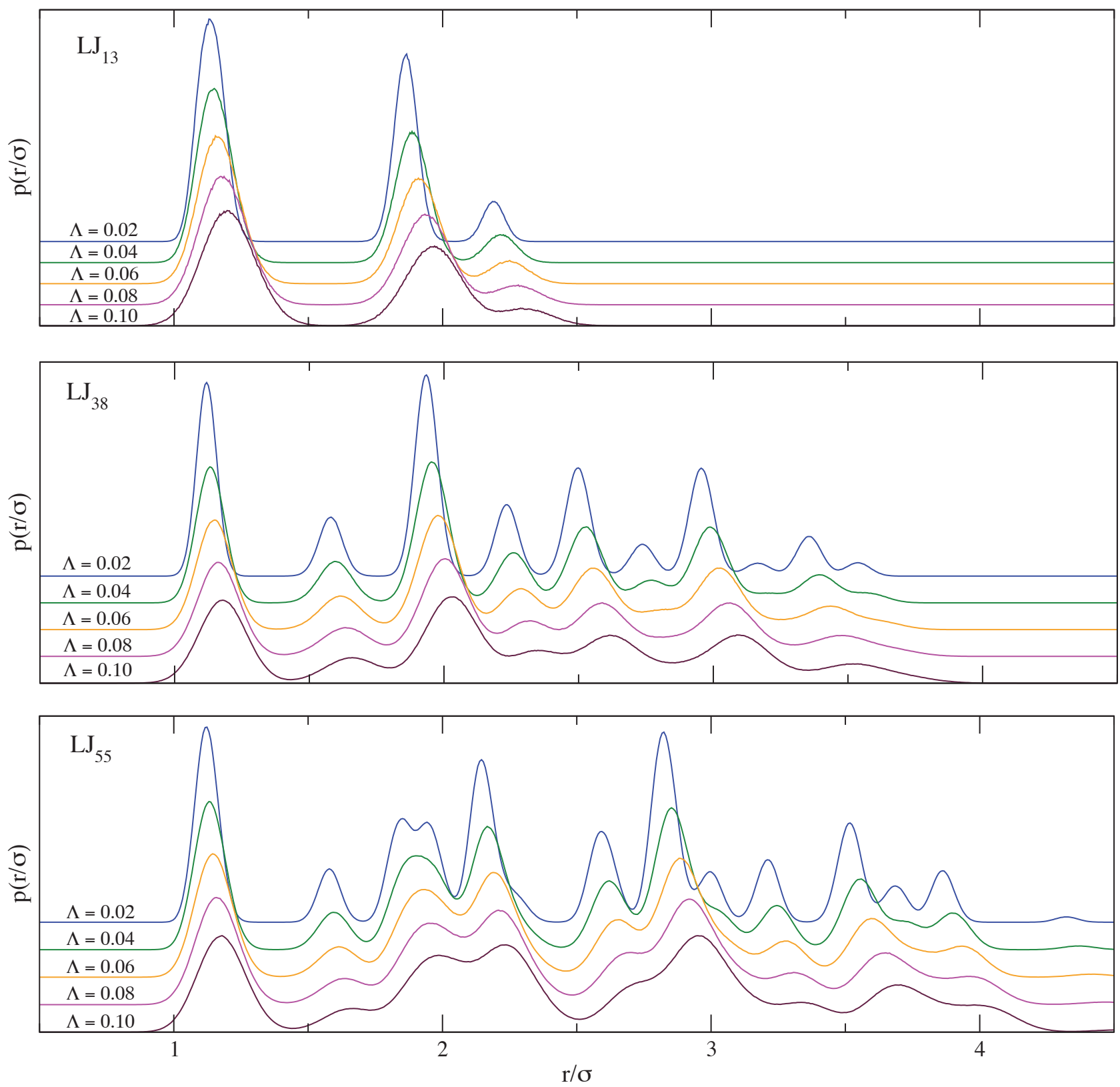

FIG. 5. Evolution of the radial distribution function for the ground states $(T=0)$ of the quantum $\mathrm{LJ}_{n}(n=13,38,55)$ clusters with quantum de Boer parameter $\Lambda=\hbar /(\sigma \sqrt{\varepsilon})$, computed using the self-consistent phonons method. The method is able to produce both the broadening and drifting of the peaks with increasing quantum character. 


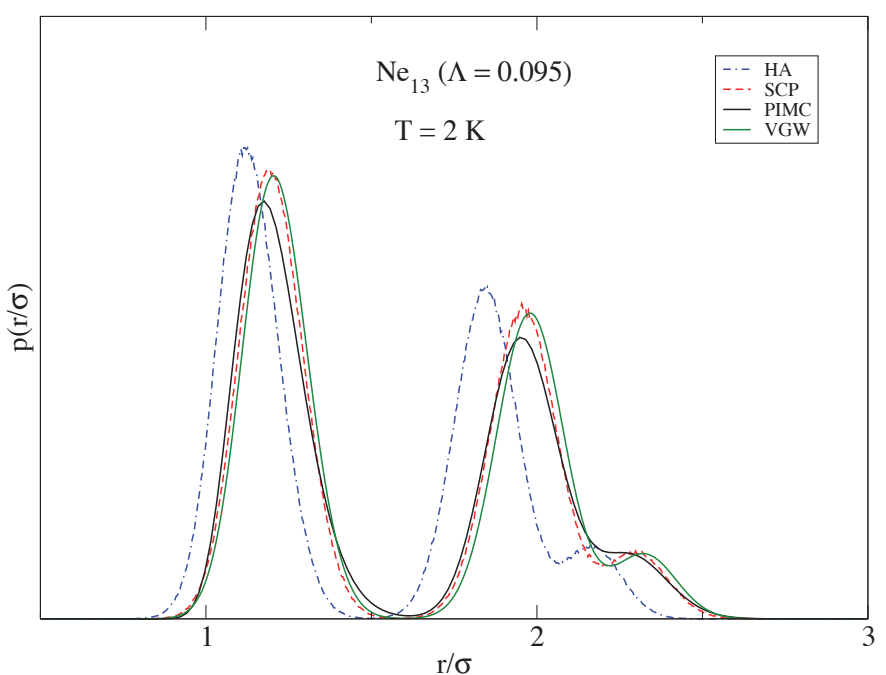

FIG. 6. Radial distribution function for the quantum $\mathrm{Ne}_{13}$ cluster at temperature $T=2 \mathrm{~K}$ (or, more precisely, an $\mathrm{LJ}_{13}$ cluster with de Boer quantum parameter $\Lambda=0.095$ at temperature $\left.T=0.056 \varepsilon / k_{B}\right)$ computed using the standard harmonic approximation (HA), self-consistent phonons (SCP) approximation, and path integral Monte Carlo (PIMC) ${ }^{33}$. 

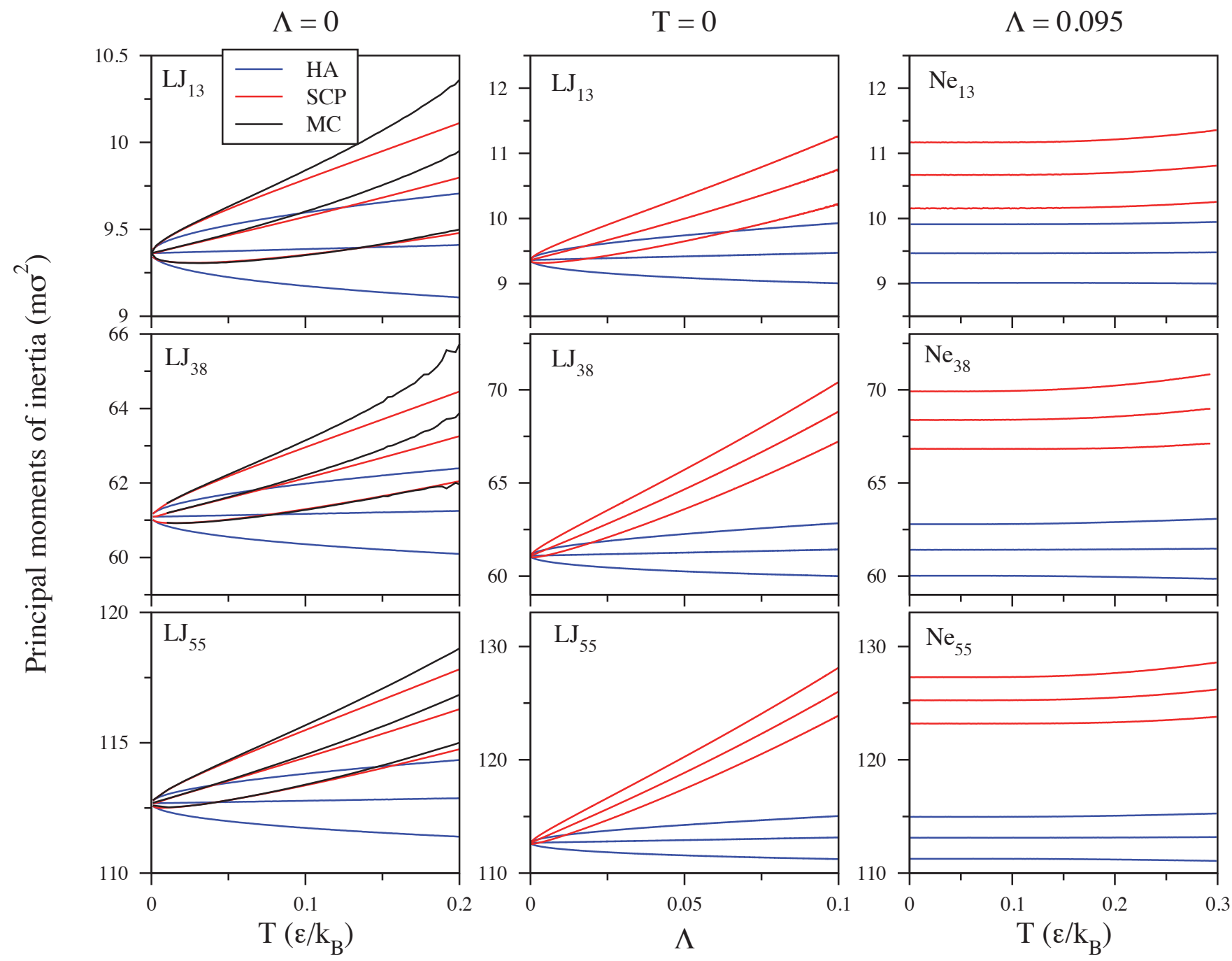

FIG. 7. Principal moments of inertia for the $\mathrm{LJ}_{13}, \mathrm{LJ}_{38}$, and $\mathrm{LJ}_{55}$ clusters for three cases: as a function of temperature for the classical case $(\Lambda=0)$ (left column), as a function of de Boer delocalization length for the quantum ground state $(T=0)$ (center column), and as a function of temperature for the quantum neon clusters $(\Lambda=0.095)$. Results were obtained using the standard harmonic approximation (HA), the self-consistent phonons (SCP) method, and Markov chain Monte Carlo (MC). In the classical case, comparison with the exact MC method reveals SCP to be significantly more accurate than the standard harmonic approximation. In all cases, the SCP results show a much stronger dependence on temperature and de Boer parameter than the HA results. 